\title{
ON CERTAIN COMPARISON THEOREMS FOR HALF-LINEAR DYNAMIC EQUATIONS ON TIME SCALES
}

\author{
PAVEL ŘEHÁK
}

Received 9 October 2002

We obtain comparison theorems for the second-order half-linear dynamic equation $\left[r(t) \Phi\left(y^{\Delta}\right)\right]^{\Delta}+p(t) \Phi\left(y^{\sigma}\right)=0$, where $\Phi(x)=|x|^{\alpha-1} \operatorname{sgn} x$ with $\alpha>1$. In particular, it is shown that the nonoscillation of the previous dynamic equation is preserved if we multiply the coefficient $p(t)$ by a suitable function $q(t)$ and lower the exponent $\alpha$ in the nonlinearity $\Phi$, under certain assumptions. Moreover, we give a generalization of HilleWintner comparison theorem. In addition to the aspect of unification and extension, our theorems provide some new results even in the continuous and the discrete case.

\section{Introduction}

In [17], it was shown that the basic results (in particular, the Reid roundabout theorem and, consequently, the Sturmian theory) known from the oscillation theory of the SturmLiouville differential equation

$$
\left(r(t) y^{\prime}\right)^{\prime}+p(t) y=0
$$

can be extended to the half-linear dynamic equation

$$
\left[r(t) \Phi\left(y^{\Delta}\right)\right]^{\Delta}+p(t) \Phi\left(y^{\sigma}\right)=0
$$

on an arbitrary time scale $\mathbb{T}$ (i.e., a closed subset of $\mathbb{R}$ ), where $r(t)$ and $p(t)$ are real rightdense continuous (rd-continuous) functions on $\mathbb{T}$ with $r(t) \neq 0$ and $\Phi(x)=|x|^{\alpha-1} \operatorname{sgn} x$ with $\alpha>1$. Moreover, in the same paper, it was proved that under the assumption of a right-dense continuity of the coefficients $r(t)$ and $p(t)$, the initial value problem involving (1.2) is uniquely solvable. The terminology half-linear is justified by the fact that the space of all solutions of (1.2) is homogeneous, but not generally additive. Thus, it has just half of the properties of a linear space. Equation (1.2) covers the half-linear differential equation $($ when $\mathbb{T}=\mathbb{R}$ )

$$
\left[r(t) \Phi\left(y^{\prime}\right)\right]^{\prime}+p(t) \Phi(y)=0
$$


and the half-linear difference equation $($ when $\mathbb{T}=\mathbb{Z})$

$$
\Delta\left[r_{k} \Phi\left(\Delta y_{k}\right)\right]+p_{k} \Phi\left(y_{k+1}\right)=0
$$

Furthermore, (1.1) is a special case of (1.3) (when $\alpha=2)$, and if $\Phi=$ id (i.e., $\alpha=2$ ), then (1.4) reduces to the Sturm-Liouville difference equation

$$
\Delta\left(r_{k} \Delta y_{k}\right)+p_{k} y_{k+1}=0
$$

Finally, the linear dynamic equation

$$
\left(r(t) y^{\Delta}\right)^{\Delta}+p(t) y^{\sigma}=0
$$

which covers (1.1) and (1.5) when $\mathbb{T}=\mathbb{R}$ and $\mathbb{T}=\mathbb{Z}$, respectively, is a special case of (1.2) (when $\alpha=2$ ). It means that the theory of (1.2) unifies and extends the theories of all mentioned equations and also explains some discrepancies between them. Note that the basic results concerning oscillatory properties of (1.1), (1.5), (1.6), (1.3), and (1.4) can be found, for example, in $[9,15,16,20],[1,2,11],[3],[5,6,13]$, and $[17,18,19]$, respectively.

The most important oscillatory properties of (1.2) are described by the so-called Reid roundabout theorem; see [17, Theorem 2]. There are several important consequences of this theorem; two of them-the Riccati technique and the Sturm-type comparison theorem (see the next section) — are used to prove our results.

In this paper, we present two types of comparison theorems. The first one actually contains two statements. First, we give a condition in terms of the inequality between the integrals $\int_{t}^{\infty} p(s) \Delta s$ and $\int_{t}^{\infty} P(s) \Delta s$ (i.e., we compare the coefficients "on average;" note that in the classical Sturm-type theorem, the coefficients are compared "pointwise"), where $P(t)$ is the corresponding coefficient to $p(t)$ of the equation which is compared with (1.2). This statement unifies and generalizes [12, Theorem 2] and [18, Theorem 4], and for historical reasons, it can be called of Hille-Wintner type. Note that in [12] (this paper concerns (1.3)), the coefficient $p(t)$ is assumed to be nonnegative. Second, we assume the condition in terms of the inequality between the exponents of the power function $\Phi$. This enables, among others, to compare a half-linear equation with a linear one. Note that, in this sense (i.e., the relation between two equations with different nonlinearities), the statement is new even in the continuous case (i.e., when $\mathbb{T}=\mathbb{R}$ ). In the proof, we combine the Riccati technique with the application of the Schauder fixed-point theorem. Our second type of comparison theorems says that, under certain additional conditions, the (non)oscillation of (1.2) is preserved when multiplying the coefficient $p(t)$ by a suitable function $q(t)$. It extends the result in [7] and its proof is based on the Riccati technique.

The paper is organized as follows. In Section 2, we give basic information concerning the calculus on time scales, some auxiliary statements including the Riccati technique, and a background for an application of the Schauder fixed-point theorem. The main results-comparison theorems-are proved in Section 3, where some comments and an example can also be found. 


\section{Preliminaries}

We start with introducing the following concepts related to the notion of time scales. It was established by Hilger in his Ph.D. dissertation in 1988; see [10]. We refer to [3] for additional details concerning the calculus on time scales. Let $\mathbb{T}$ be a time scale (i.e., a closed subset of $\mathbb{R}$ ). We assume throughout that $\mathbb{T}$ has the topology that it inherits from the standard topology on the real numbers $\mathbb{R}$. Because of the character of our result, we suppose that $\sup \mathbb{T}=\infty$. Define the forward jump operator $\sigma(t)$ at $t \in \mathbb{T}$ by $\sigma(t):=$ $\inf \{\tau>t: \tau \in \mathbb{T}\}$, and the backward jump operator $\rho(t)$ at $t \in \mathbb{T}$ by $\rho(t):=\sup \{\tau<t$ : $\tau \in \mathbb{T}\}$. If $\sigma(t)>t$, we say $t$ is right-scattered, while if $\rho(t)<t$, we say $t$ is left-scattered. If $\sigma(t)=t$, we say $t$ is right-dense, while if $\rho(t)=t$, we say $t$ is left-dense. We will also use the notation $\mu(t):=\sigma(t)-t$ which is called the graininess function. A function $f: \mathbb{T} \rightarrow \mathbb{R}$ is called (delta) differentiable at $t \in \mathbb{T}$ with (delta) derivative $f^{\Delta}(t) \in \mathbb{R}$ if there exists the (finite) limit

$$
f^{\Delta}(t):=\lim _{s \rightarrow t, \sigma(s) \neq t} \frac{f(\sigma(s))-f(t)}{\sigma(s)-t} .
$$

We use the notation $f^{\sigma}(t)=f(\sigma(t))$ for $t \in \mathbb{T}$, that is, $f^{\sigma}=f \circ \sigma$. The notations $[a, b]$, $[a, b),[a, \infty)$, and so forth denote time scales intervals. A function $f: \mathbb{T} \rightarrow \mathbb{R}$ is said to be $r d$-continuous provided that $f$ is continuous at right-dense points in $\mathbb{T}$ and at left-dense points in $\mathbb{T}$, left-hand limits exist and are finite. We write $f \in C_{\mathrm{rd}}(\mathbb{T})$. The integral of a rd-continuous function $f$ (it indeed exists) is defined by means of the antiderivative $F$, that is, $\int_{a}^{b} f(t) \Delta t=F(b)-F(a)$, where $F$ is such that $F^{\Delta}=f$.

We say that a solution $y$ of (1.2) has a generalized zero at $t$ in case $y(t)=0$. We say $y$ has a generalized zero in $(t, \sigma(t))$ in case $r(t) y(t) y(\sigma(t))<0$ and $\mu(t)>0$. We say that $(1.2)$ is disconjugate on the interval $[a, b]$ if there is no nontrivial solution of (1.2) with two (or more) generalized zeros in $[a, b]$.

Equation (1.2) is said to be nonoscillatory (on $[a, \infty)$ ) if there exists $c \in[a, \infty)$ such that this equation is disconjugate on $[c, d]$ for every $d>c$. In the opposite case, $(1.2)$ is said to be oscillatory (on $[a, \infty)$ ). Oscillation of (1.2) may be equivalently defined as follows. A nontrivial solution $y$ of (1.2) is called oscillatory if it has infinitely many (isolated) generalized zeros in $[a, \infty)$. By the Sturm-type separation theorem, see [17, Theorem 3], one solution of (1.2) is (non)oscillatory if and only if every solution of (1.2) is (non)oscillatory. Hence, we can speak about oscillation or nonoscillation of (1.2).

The classical Sturm's result can be generalized as follows, see [17, Theorem 3].

Proposition 2.1 (Sturm (or Sturm-Picone)-type comparison theorem). Consider the equation

$$
\left[R(t) \Phi\left(y^{\Delta}\right)\right]^{\Delta}+P(t) \Phi\left(y^{\sigma}\right)=0
$$

where $R$ and $P$ satisfy the same assumptions as $r$ and $p$. Suppose that $r(t) \leq R(t)$ and $P(t) \leq p(t)$ on $[T, \infty)$ for all large $T$. Then (1.2) is nonoscillatory implying that (2.2) is nonoscillatory. 
554 Comparison theorems for half-linear dynamic equations

Along with (1.2) (defined on a time scale interval of the form $[a, \infty)$ ), consider the generalized Riccati dynamic equation

$$
\mathscr{R}[w]:=w^{\Delta}+p(t)+\mathscr{S}[w, r ; \alpha](t)=0,
$$

where

$$
\mathscr{S}[w, r ; \alpha]=\lim _{\lambda \rightarrow \mu} \frac{w}{\lambda}\left(1-\frac{r}{\Phi\left(\Phi^{-1}(r)+\lambda \Phi^{-1}(w)\right)}\right) .
$$

As we will see, it is related to the original equation by the Riccati-type substitution $w(t)=$ $r(t) \Phi\left[y^{\Delta}(t) / y(t)\right]$. Observe that

$$
\mathscr{S}[w, r ; \alpha](t)= \begin{cases}\left\{\frac{(\alpha-1)}{\Phi^{-1}(r)}|w|^{\beta}\right\}(t) & \text { for right-dense } t, \\ \left\{\frac{w}{\mu}\left(1-\frac{r}{\Phi\left(\Phi^{-1}(r)+\mu \Phi^{-1}(w)\right)}\right)\right\}(t) & \text { for right-scattered } t,\end{cases}
$$

where the l'Hôpital's rule is used in the first case, $\Phi^{-1}$ denotes the inverse of $\Phi$ (i.e., $\Phi^{-1}(x)=|x|^{\beta-1} \operatorname{sgn} x$ ), and $\beta$ is the conjugate number of $\alpha$ (i.e., $1 / \alpha+1 / \beta=1$ ).

The proof of the following statement is based on the Reid roundabout theorem and Sturm-type comparison theorem (see [17, Lemma 14]), and it is usually referred to as the Riccati technique.

Proposition 2.2 (Riccati technique). Equation (1.2) is nonoscillatory if and only if there exists $T \in[a, \infty)$ and a function $w$ satisfying the generalized Riccati dynamic inequality $\mathscr{R}[w](t) \leq 0$ with $\left\{\Phi^{-1}(r)+\mu \Phi^{-1}(w)\right\}(t)>0$ for $t \in[T, \infty)$.

A behavior of the operator $\mathscr{S}$ with respect to its arguments will be described by the properties of the function

$$
S(x, y, \alpha)=\lim _{\lambda \rightarrow \mu} \frac{x}{\lambda}\left(1-\frac{y}{\Phi\left(\Phi^{-1}(y)+\lambda \Phi^{-1}(x)\right)}\right) .
$$

Note that the function $S$ can be understood as a "half-linear generalization" of the function $x^{2} /(y+\mu x)$ that corresponds to the operator occurring in the Riccati dynamic equation associated to linear dynamic equation (1.6), and hence a similar behavior of these functions can be expected in a certain sense.

Lemma 2.3. The function $S$ has the following properties:

(i) let $y>0$, then $x(\partial S / \partial x)(x, y, \alpha) \geq 0$ for $\Phi^{-1}(y)+\mu \Phi^{-1}(x)>0$, where $(\partial S / \partial x)(x, y, \alpha)$ $=0$ if and only if $x=0$;

(ii) $S(x, y, \alpha) \geq 0$ for $\Phi^{-1}(y)+\mu \Phi^{-1}(x)>0$, where the equality holds if and only if $x=0$;

(iii) if $x>0, y>0$, and

$$
\gamma:=\lim _{\lambda \rightarrow \mu} \frac{(1+\lambda z) \ln (1+\lambda z)-\lambda z \ln z}{\lambda} \geq 0,
$$

where $z:=(x / y)^{1 /(\alpha-1)}$, then $(\partial S / \partial \alpha)(x, y, \alpha) \geq 0$. 
Remark 2.4. (i) Using the L'Hôpital's rule, we have $\gamma=z-z \ln z$ when $\mu=0$.

(ii) It is easy to see that if $\mu \geq 1$, then $\gamma \geq 0$, since

$$
\gamma=\frac{\ln (1+\mu z)+\mu z \ln ((1+\mu z) / z)}{\mu} .
$$

On the other hand, if $\mu \in[0,1$ ), then $z$ being small (more precisely, $z \leq 1$, but in fact, the right-hand side may be greater than 1 ; it depends on $\mu$ ) is a sufficient condition for $\gamma$ to be nonnegative. We notice how the graininess function plays the role in the monotone nature of $S$. Observe that $S$ is not always nondecreasing with respect to $\alpha$, even when $x, y>0$.

(iii) In view of the last remark, if, for example, $w(t)>0, r(t)>0, \lim _{t \rightarrow \infty} w(t)=0$, and $\liminf _{t \rightarrow \infty} r(t)>0$, then $\partial \mathscr{S}(w(t), r(t) ; \alpha) / \partial \alpha \geq 0$ for large $t$. It is clear that the last two conditions may be dropped when $\mu(t) \geq 1$ eventually.

Proof. For the proof of (i) and (ii), see [17, Lemma 13].

To prove the property (iii), first note that for $\mu>0$, the function $S$ can be rewritten as

$$
S(x, y, \alpha)=\frac{x}{\mu}\left[1-\left(1+\mu\left(\frac{x}{y}\right)^{1 /(\alpha-1)}\right)^{1-\alpha}\right],
$$

while for $\mu=0$ it takes the form $S(x, y, \alpha)=(\alpha-1) x(x / y)^{1 /(\alpha-1)}$. Differentiating $S$ with respect to $\alpha$, using the known rules, we get

$$
\frac{\partial S}{\partial \alpha}=\frac{x}{\mu}(1+\mu z)^{-\alpha}[(1+\mu z) \ln (1+\mu z)-\mu z \ln z]
$$

in case $\mu>0$. If $\mu=0$, then we obtain $\partial S / \partial \alpha=x(z-z \ln z)$. In view of the assumptions of the lemma, Remark 2.4(i), and the equality

$$
\begin{aligned}
\lim _{\lambda \rightarrow \mu} \frac{\partial}{\partial \alpha}\left\{\frac{x}{\lambda}\left[1-\left(1+\lambda\left(\frac{x}{y}\right)^{1 /(\alpha-1)}\right)^{1-\alpha}\right]\right\} \\
=\frac{\partial}{\partial \alpha}\left\{\lim _{\lambda \rightarrow \mu} \frac{x}{\lambda}\left[1-\left(1+\lambda\left(\frac{x}{y}\right)^{1 /(\alpha-1)}\right)^{1-\alpha}\right]\right\},
\end{aligned}
$$

we get the statement.

The next lemma claims that, under certain assumptions, an eventually positive solution of (nonoscillatory) equation (1.2) has an eventually positive delta-derivative, consequently, (2.3) has a positive solution.

Lemma 2.5. Assume that $r(t)>0$,

$$
\liminf _{t \rightarrow \infty} \int_{T}^{t} p(s) \Delta s \geq 0, \quad \liminf _{t \rightarrow \infty} \int_{T}^{t} p(s) \Delta s \equiv 0,
$$


556 Comparison theorems for half-linear dynamic equations

for all large $T$, and

$$
\int_{a}^{\infty} r^{1-\beta}(s) \Delta s=\infty
$$

If $y$ is a solution of (1.2) such that $y(t)>0$ for $t \in[T, \infty)$, then there exists $T_{1} \in[T, \infty)$ such that $y^{\Delta}(t)>0$ for $t \in\left[T_{1}, \infty\right)$.

Proof. The proof is by contradiction. We consider the following two cases.

Case 1. Suppose that $y^{\Delta}(t)<0$ for $t \in[T, \infty)$. Then also $[\Phi(y)]^{\Delta}(t)<0$ for $t \in[T, \infty)$ since

$$
[\Phi(y)]^{\Delta}(t)=\frac{d}{d y} \Phi[y(\xi)] y^{\Delta}(t)=(\alpha-1)|y(\xi)|^{\alpha-2} y^{\Delta}(t)<0
$$

by [3, Theorem 1.87], where $t \leq \xi \leq \sigma(t)$. Another argument for $[\Phi(y)]^{\Delta}(t)<0$ is that if $y$ is decreasing, then $\Phi(y)$ is decreasing as well because of the properties of the function $\Phi$. Without loss of generality we may assume that $T$ is such that $\int_{T}^{t} p(s) \Delta s \geq 0, t \in[T, \infty)$, reasoning as in [7, Proof of Lemma 13]. Define $Q(t, T)=\int_{T}^{t} p(s) \Delta s$. The integration by parts gives

$$
\begin{aligned}
\int_{T}^{t} p(s) \Phi\left[y^{\sigma}(s)\right] \Delta s & =\int_{T}^{t} Q^{\Delta}(s, T) \Phi\left(y^{\sigma}(s)\right) \Delta s \\
& =Q(t, T) \Phi(y(t))-\int_{T}^{t} Q(s, T)[\Phi(y(s))]^{\Delta} \Delta s \geq 0 .
\end{aligned}
$$

Integrating (1.2), we have, using the last estimate,

$$
r(t) \Phi\left(y^{\Delta}(t)\right)-r(T) \Phi\left(y^{\Delta}(T)\right)=\int_{T}^{t}\left[r(s) \Phi\left(y^{\Delta}(s)\right)\right]^{\Delta} \Delta s \leq 0 .
$$

Hence,

$$
y^{\Delta}(t) \leq \frac{r^{\beta-1}(T) y^{\Delta}(T)}{r^{\beta-1}(t)}
$$

for $t \in[T, \infty)$. Integrating (2.17) for $t \geq T$, we see that $y(t) \rightarrow-\infty$ by (2.13), a contradiction. Therefore, $y^{\Delta}(t)<0$ cannot hold for all large $t$.

Case 2. Next, if $y^{\Delta}(t) \ngtr 0$ eventually, then for every (large) $T \in[a, \infty)$, there exists $T_{0} \in$ $[T, \infty)$ such that $y^{\Delta}\left(T_{0}\right) \leq 0$ and we may suppose that $\liminf _{t \rightarrow \infty} \int_{T}^{t} p(s) \Delta s \geq 0$. Since $y(t)>0$ for $t \in[T, \infty)$, the function $w(t)=r(t) \Phi\left[y^{\Delta}(t) / y(t)\right]$ satisfies the generalized Riccati equation (2.3) with $\left\{\Phi^{-1}(r)+\mu \Phi^{-1}(w)\right\}(t)>0$ for $t \in[T, \infty)$. Integrating (2.3) from $T_{0}$ to $t, t \geq T_{0}$, gives

$$
w(t)=w\left(T_{0}\right)-\int_{T_{0}}^{t} p(s) \Delta s-\int_{T_{0}}^{t} \mathscr{S}(w, r ; \alpha)(s) \Delta s .
$$


Therefore, it follows that limsup $\operatorname{sum}_{t \rightarrow \infty} w(t)<0$, using the facts $w\left(T_{0}\right) \leq 0, w(t)$ is eventually nontrivial, and (2.12) holds. Indeed, there is $M>0$ such that $\int_{T_{0}}^{t} \mathscr{Y}(w, r ; \alpha)(s) \Delta s \geq M$ and $\int_{T_{0}}^{t} p(s) \Delta s \geq-M / 2$ for all large $t$. Hence, there exists $T_{1} \in[T, \infty)$ such that $w(t)<0$ for $t \in\left[T_{1}, \infty\right)$ and so $y^{\Delta}(t)<0$ for $t \in\left[T_{1}, \infty\right)$, a contradiction to the first part.

In the next lemma, a necessary condition for the nonoscillation of (1.2) is given in terms of solvability of generalized Riccati integral inequality under certain assumptions. Note that a closer examination of the proof of Theorem 3.1 shows that this condition is also sufficient.

LEMMA 2.6. Let the assumptions of Lemma 2.5 hold and assume further that $\int_{a}^{\infty} p(s) \Delta s=$ $\lim _{t \rightarrow \infty} \int_{a}^{t} p(s) \Delta s$ is convergent. Let $y(t)$ be a solution of (1.2) such that $y(t)>0$ for $t \in$ $[T, \infty)$. Then there exists $T_{1} \in[T, \infty)$ such that

$$
w(t) \geq \int_{t}^{\infty} p(s) \Delta s+\int_{t}^{\infty} \mathscr{S}(w, r ; \alpha)(s) \Delta s
$$

for $t \in\left[T_{1}, \infty\right)$, where $w(t)=r(t) \Phi\left[y^{\Delta}(t) / y(t)\right]>0$.

Proof. From Lemma 2.5, there exists $T_{1} \in[T, \infty)$ such that $w(t)>0$ for $t \in\left[T_{1}, \infty\right)$ and $w$ satisfies (2.3) for $t \in[T, \infty)$ (clearly, with $\left.\left\{\Phi^{-1}(r)+\mu \Phi^{-1}(w)\right\}(t)>0\right)$. Integrating (2.3) from $t$ to $s, s \geq t \geq T_{1}$, we get

$$
w(s)-w(t)+\int_{t}^{s} p(\xi) \Delta \xi+\int_{t}^{s} \mathscr{S}(w, r ; \alpha)(\xi) \Delta \xi=0
$$

Therefore,

$$
0<w(s) \leq w(t)-\int_{t}^{s} p(\xi) \Delta \xi
$$

and hence,

$$
w(t) \geq \int_{t}^{s} p(\xi) \Delta \xi+\int_{t}^{s} \mathscr{S}(w, r ; \alpha)(\xi) \Delta \xi
$$

for $s \geq t \geq T_{1}$. Letting $s \rightarrow \infty$, we obtain (2.19).

In the last part of this section, we give a background for the application of the Schauder fixed-point theorem. It will be used in the proof of Theorem 3.1. We start by recalling the Schauder theorem that is applicable for our setting in dynamic equations.

Proposition 2.7 (Schauder fixed-point theorem, [8, Theorem 6.44]). Let $\mathcal{N}$ be a normed space and $X$ be a nonempty, closed, convex subset of $\mathcal{N}$. If $\mathcal{T}$ is a continuous mapping such that $\mathscr{T}(X) \subseteq X$ (i.e., mapping $X$ into itself) and $\mathscr{T}(X)$ is relatively compact, then $\mathcal{T}$ has a fixed point in $X$.

Denote with $C_{T S}^{B}[a, \infty)$ the linear space of all continuous functions $f:[a, \infty) \rightarrow \mathbb{R}$ such that $\sup _{t \in[a, \infty)}|f(t)|<\infty$. Define this supremum to be the norm $\|f\|=\sup _{t \in[a, \infty)}|f(t)|$. 
The following statement can be understood as a time scale version of the Arzelà-Ascoli theorem. For the discrete analog of this well-known theorem, see [4, Theorem 3.3]. Note that for $\mathbb{T}=\mathbb{N}$ we get $C_{T S}^{B}=\ell^{\infty}$, and condition (ii) in the next lemma holds trivially.

Lemma 2.8. Let $X$ be a subset of $C_{T S}^{B}[a, \infty)$ having the following properties:

(i) $X$ is bounded;

(ii) on every compact subinterval $J$ of $[a, \infty)$, there exists, for any $\varepsilon>0, \delta>0$ such that $t_{1}, t_{2} \in J,\left|t_{1}-t_{2}\right|<\delta$ implies $\left|f\left(t_{1}\right)-f\left(t_{2}\right)\right|<\varepsilon$ for all $f \in X$ (i.e., the functions of $X$ are locally equicontinuous);

(iii) for every $\varepsilon>0$, there exists $b \in[a, \infty)$ such that $t_{1}, t_{2} \in[b, \infty)$ implies $\left|f\left(t_{1}\right)-f\left(t_{2}\right)\right|$ $<\varepsilon$ for all $f \in X$ (in the "discrete terminology," $X$ is said to be uniformly Cauchy).

Then $X$ is relative compact.

Proof. By [8, Theorem 6.33], it is sufficient to construct a finite $\varepsilon$-net for any $\varepsilon$. Since the proof is more or less obvious, we mention just some of its important points and omit details. In view of the properties (i), (ii), and (iii), it is possible to construct a twodimensional grid, where the vertical values are the elements $y_{1}, \ldots, y_{m} \in \mathbb{R},-K=y_{1}<$ $y_{2}<\cdots<y_{m}=K, K$ being such that $\|f\| \leq K$ for all $f \in X$, and sufficiently close to neighbors, that is, $y_{i+1}-y_{i}$ is a sufficiently small number depending on $\varepsilon$. The horizontal values $x_{1}, \ldots, x_{m} \in \mathbb{T}, a=x_{1}<x_{2}<\cdots<x_{n}=b$, are sufficiently close to their neighbors in the sense that if they are close to dense points, the differences of the values of $f(f \in X)$ at these points are small_-depend on $\varepsilon$ (this is possible thanks to the local equicontinuity) or they are isolated and sufficiently far from each other; $b \geq a$ being such that $\mid f\left(t_{1}\right)-$ $f\left(t_{2}\right) \mid$ is sufficiently small (depends on $\varepsilon$ ) whenever $t_{1}, t_{2} \in[b, \infty)$ for all $f \in X$. Such $b$ exists thanks to the property (iii). Now, having such grid for any $f \in X$, we can construct a linear fractional function $g$ which approximate $f$ (in fact, $\|f-g\|<\varepsilon$ ). The number of functions $g$ constructed in this way is finite and thus the set of such functions forms a finite $\mathcal{E}$-net for $X$.

\section{Main results}

We start with Hille-Wintner-type comparison theorem involving also the condition in terms of the change of the exponents in the power function $\Phi$. Along with (1.2), consider the equation

$$
\left[R(t) \Phi_{\bar{\alpha}}\left(x^{\Delta}\right)\right]^{\Delta}+P(t) \Phi_{\bar{\alpha}}\left(x^{\sigma}\right)=0
$$

where $R$ and $P$ satisfy the same assumptions as $r$ and $p$, and $\Phi_{\bar{\alpha}}(x)=|x|^{\bar{\alpha}-1} \operatorname{sgn} x$ with $\bar{\alpha}>1$.

Theorem 3.1. Assume $0<R(t) \leq r(t)$,

$$
0 \leq \int_{t}^{\infty} p(s) \Delta s \leq \int_{t}^{\infty} P(s) \Delta s
$$


for all large $t$ (in particular, these integrals exist as finite numbers),

$$
\int_{a}^{\infty} R^{1-\bar{\beta}}(s) \Delta s=\infty
$$

with $\bar{\beta}>1$ being the conjugate number to $\bar{\alpha}$, and $1<\alpha \leq \bar{\alpha}$. Further, suppose that $\liminf _{t \rightarrow \infty} R(t)>0$ when $\mu(t) \nsucceq 1$ eventually (if $\mu(t) \geq 1$ eventually, then this condition may be dropped-see also Remark 2.4). If (3.1) is nonoscillatory, then so is (1.2).

Proof. By Lemma 2.6, the assumptions of the theorem imply the existence of a function $z$ (actually, $z=R \Phi_{\bar{\alpha}}\left(x^{\Delta} / x\right), x$ being an eventually positive increasing solution of (3.1)) and $T \in[a, \infty)$ such that

$$
z(t) \geq \int_{t}^{\infty} P(s) \Delta s+\int_{t}^{\infty} \mathscr{Y}(z(s), R(s) ; \bar{\alpha}) \Delta s=: Z(t)
$$

with $z(t)>0$ for $t \geq T$. Without loss of generality, we may assume that (3.2) holds for $t \geq T$. Define the set $\Omega=\left\{w \in C_{T S}^{B}[T, \infty): 0 \leq w(t) \leq Z(t)\right.$ for $\left.t \geq T\right\}$ and the operator $\mathscr{T}: \Omega \rightarrow C_{T S}^{B}[T, \infty)$ defined by

$$
\mathscr{T}(w)(t)=\int_{t}^{\infty} p(s) \Delta s+\int_{t}^{\infty} \mathscr{Y}(w(s), R(s) ; \alpha) \Delta s
$$

for $w \in \mathscr{T}$. In view of the assumptions of the theorem and the properties of $\mathscr{T}$, the operator $\mathcal{T}$ is well defined. It is very easy to see that $\Omega$ is closed and convex.

We show that $\mathscr{T}$ maps $\Omega$ into itself. Suppose that $w \in \Omega$ and define $v(t)=\mathscr{T}(w)(t)$, $t \geq T$. Obviously, $v(t) \geq 0$ for $t \geq T$. We prove that $v(t) \leq Z(t)$. First note that since $w \in \Omega$ is small for large $t$ and $\liminf _{t \rightarrow \infty} R(t)>0$ (provided that $\mu(t) \nsucceq 1$ eventually), we have $w(t) / R(t) \leq 1$ for large $t$ (without loss of generality, we may suppose that $T$ is such that $Z(t) / R(t) \leq 1$ for $t \geq T$ in case $\mu(t) \nsupseteq 1$ eventually), and so the assumptions of Lemma 2.3(iii) are satisfied (see also Remark 2.4). Now we get

$$
\begin{aligned}
v(t) & =\int_{t}^{\infty} p(s) \Delta s+\int_{t}^{\infty} \mathscr{Y}(w(s), R(s) ; \alpha) \Delta s \\
& \leq \int_{t}^{\infty} P(s) \Delta s+\int_{t}^{\infty} \mathscr{Y}(w(s), R(s) ; \alpha) \Delta s \\
& \leq \int_{t}^{\infty} P(s) \Delta s+\int_{t}^{\infty} \mathscr{Y}(w(s), R(s) ; \bar{\alpha}) \Delta s \leq Z(t)
\end{aligned}
$$

by the assumptions of the theorem and by Lemma 2.3. Hence $\mathscr{T}(\Omega) \subset \Omega$.

According to Lemma 2.8, to prove the relative compactness of $\mathscr{T}(\Omega)$, it is sufficient to verify that conditions (i), (ii), and (iii) hold for $\mathscr{T}(\Omega)$. Clearly, $\mathscr{T}(\Omega) \subset \Omega$ implies the boundedness of $\mathscr{T}(\Omega)$. In view of the definition of $\mathscr{T}$, for any $w \in \Omega$, we have $0 \leq$ $-(\mathscr{T}(w))^{\Delta}(t)=p(t)+\mathscr{S}(w(t), R(t) ; \alpha) \leq p(t)+\mathscr{S}(z(t), R(t) ; \bar{\alpha})$, which proves the equicontinuity of the elements of $\mathscr{T}(\Omega)$. Finally, we verify that $\mathscr{T}(\Omega)$ is "uniformly Cauchy." 
Let $\varepsilon>0$ be given. We have to show that there exists $t_{0} \in[T, \infty)$ such that for any $t_{1}, t_{2} \in$ $\left[t_{0}, \infty\right)$, it holds that $\left|\mathcal{T}(w)\left(t_{1}\right)-\mathscr{T}(w)\left(t_{2}\right)\right|<\varepsilon$ for any $w \in \Omega$. Without loss of generality, suppose that $t_{1}<t_{2}$. Then we have

$$
\left|\mathscr{T}(w)\left(t_{1}\right)-\mathscr{T}(w)\left(t_{2}\right)\right| \leq\left|\int_{t_{1}}^{t_{2}} p(s) \Delta s\right|+\int_{t_{1}}^{t_{2}} \mathscr{S}(w(s), R(s) ; \alpha) \Delta s
$$

Since the integrals in (3.7) are convergent, for any $\varepsilon>0$, one can find $t_{0} \in[T, \infty)$ such that

$$
\left|\int_{t_{1}}^{t_{2}} p(s) \Delta s\right|<\frac{\varepsilon}{2}, \quad \int_{t_{1}}^{t_{2}} \mathscr{S}(w(s), R(s) ; \alpha) \Delta s<\frac{\varepsilon}{2}
$$

whenever $t_{2}>t_{1} \geq t_{0}$. From here and (3.7), we get the desired inequality. Hence, $\mathscr{T}(\Omega)$ is relatively compact.

The last hypothesis, which has to be verified, is the continuity of $\mathscr{T}$ in $\Omega$. Let $\left\{w_{n}\right\}$, $n \in \mathbb{N}$, be a sequence in $\Omega$ which uniformly converges on every compact subinterval of $[T, \infty)$ to $\bar{w} \in \Omega$. Because $\mathscr{T}(\Omega)$ is relatively compact, the sequence $\left\{\mathscr{T}\left(w_{n}\right)\right\}$ admits a subsequence $\left\{\mathscr{T}\left(w_{n_{j}}\right)\right\}$ converging in the topology of $C_{T S}^{B}[T, \infty)$ to $\bar{v}$. The inequality $\mathscr{Y}\left(w_{n_{j}}(t), R(t) ; \alpha\right) \leq \mathscr{Y}(z(t), R(t) ; \bar{\alpha})$ implies that the integral $\int_{t}^{\infty} \mathscr{Y}\left(w_{n_{j}}(s), R(s) ; \alpha\right) \Delta s$ is totally convergent. Hence, by the Lebesgue dominated convergence theorem on time scales, see [14], the sequence $\left\{\mathscr{T}\left(w_{n_{j}}\right)\right\}$ converges to $\mathscr{T}(\bar{w})$. In view of the uniqueness of the limit, $\mathscr{T}(\bar{w})=\bar{v}$ is the only cluster point of the sequence $\left\{\mathscr{T}\left(w_{n}\right)\right\}$ that proves the continuity of $\mathcal{T}$ in $\Omega$.

Therefore, it follows from Proposition 2.7 that there exists an element $w \in \Omega$ such that $\mathscr{T}(w)=w$. In view of how the operator $\mathscr{T}$ is defined, this (positive) function $w$ satisfies the equation

$$
w(t)=\int_{t}^{\infty} p(s) \Delta s+\int_{t}^{\infty} \mathscr{S}(w(s), R(s) ; \alpha) \Delta s
$$

$t \geq T$, and hence, also the equation $w^{\Delta}+p(t)+\mathscr{S}(w, R ; \alpha)(t)=0$, clearly, with $\Phi^{-1}(R)+$ $\mu \Phi^{-1}(w)>0$. Consequently, the function $y$, given by

$$
y(T)=A \neq 0, \quad y^{\Delta}=\left(\frac{w(t)}{R(t)}\right)^{\beta-1} y
$$

$t \geq T$, is a nonoscillatory solution of $\left[R(t) \Phi\left(y^{\Delta}\right)\right]^{\Delta}+p(t) \Phi\left(y^{\sigma}\right)=0$, and hence, this equation is nonoscillatory. The statement now follows from Proposition 2.1.

Remark 3.2. (i) A closer examination of the previous proof shows that the necessary condition for nonoscillation of (1.2) in Lemma 2.6 is also sufficient.

(ii) It is not difficult to make the following observation. If (2.13) holds, $p(t) \geq 0$ (and eventually nontrivial) for all large $t, \int_{a}^{\infty} p(s) \Delta s$ converges, and (1.2) has a positive solution $y$, then the nonnegative function $w(t)=r(t) \Phi\left[y^{\Delta} / y\right]$ (in fact, it is a solution of (2.3)) is eventually nonincreasing and converges to zero. Moreover, it satisfies the integral 
equation

$$
w(t)=\int_{t}^{\infty} p(s) \Delta s+\int_{t}^{\infty} \mathscr{S}(w(s), r(s) ; \alpha) \Delta s
$$

Clearly, the solvability of (3.11) is also a sufficient condition for the nonoscillation of (1.2). In this case, it is possible to prove the comparison theorem involving the condition in terms of the inequality between exponents of the nonlinearities immediately by the Riccati technique without using the Schauder fixed-point theorem. Indeed, we have $0=w^{\Delta}(t)+p(t)+\mathscr{S}[w, r ; \alpha] \geq w^{\Delta}(t)+p(t)+\mathscr{S}[w, r ; \bar{\alpha}]$ for large $t$ provided that $\alpha \geq$ $\bar{\alpha}>1$ and $\liminf _{t \rightarrow \infty} r(t)>0$ (when $\mu(t) \nsupseteq 1$ eventually). The fact that $\left[r(t) \Phi_{\bar{\alpha}}\left(y^{\Delta}\right)\right]^{\Delta}+$ $p(t) \Phi_{\bar{\alpha}}\left(y^{\sigma}\right)=0$ is nonoscillatory then follows from Proposition 2.2. In view of Remark 2.4, the statement can be proved in this way even under the assumptions of Lemma 2.5, provided that $\mu(t) \geq 1$ eventually, since we do not need a solution of (2.3) to be close to zero. In particular, this is satisfied in the discrete case, that is, $\mathbb{T}=\mathbb{Z}$.

Now, we mention a few background details which serve to motivate our second main result. Along with (1.2), consider the equation

$$
\left[r(t) \Phi\left(y^{\Delta}\right)\right]^{\Delta}+\lambda p(t) \Phi\left(y^{\sigma}\right)=0
$$

where $\lambda$ is a real constant, and assume that $r(t)>0$. We claim that if (1.2) is nonoscillatory and $0<\lambda \leq 1$, then (3.12) is also nonoscillatory. If $p(t) \geq 0$, then this statement follows immediately from the Sturm comparison theorem (Proposition 2.1). If $p(t)$ may change sign, then dividing (3.12) by $\lambda$, we obtain an equivalent equation which is nonoscillatory again by the Sturm theorem. This can be analogously done for oscillatory counterparts. If the constant $\lambda$ is replaced by a function $q(t)$, then the situation is not so easy (when $p(t)$ may change sign; otherwise the Sturm theorem can be applied immediately). The following statements give an answer to the question "what are the conditions which guarantee that the (non)oscillation of (1.2) is preserved when multiplying the coefficient $p(t)$ by a function $q(t)$ ?" They generalize [7, Theorem 7, Corollary 8]. Along with (1.2), consider the equation

$$
\left[R(t) \Phi\left(x^{\Delta}\right)\right]^{\Delta}+q(t) P(t) \Phi\left(x^{\sigma}\right)=0
$$

where $R$ and $P$ satisfy the same assumptions as $r$ and $p$.

Theorem 3.3. Assume that $q(t) \in C_{\mathrm{rd}}^{1}[a, \infty), 0<r(t) \leq R(t), P(t) \leq p(t), 0<q(t) \leq 1$, and $q^{\Delta}(t) \leq 0$. Further, let (2.12) and (2.13) hold. Then (1.2) is nonoscillatory implying that (3.13) is nonoscillatory.

Proof. The assumptions of the theorem imply that there exists a solution $y$ of (1.2) and $T \in[a, \infty)$ such that $y(t)>0$ and $y^{\Delta}(t)>0$ on $[T, \infty)$ by Lemma 2.5. Therefore, the function $w(t):=r(t) \Phi\left(y^{\Delta}(t) / y(t)\right)>0$ satisfies (2.3) with $\left\{\Phi^{-1}(r)+\mu \Phi^{-1}(w)\right\}(t)>0$ 
562 Comparison theorems for half-linear dynamic equations

on $[T, \infty)$. We have

$$
\begin{aligned}
q \mathscr{S}[w, r ; \alpha] & =\lim _{\lambda \rightarrow \mu} \frac{w q}{\lambda}\left(1-\frac{r q}{\Phi\left[\Phi^{-1}(q) \Phi^{-1}(r)+\lambda \Phi^{-1}(q) \Phi^{-1}(w)\right]}\right) \\
& =\mathscr{S}[q w, q r ; \alpha] .
\end{aligned}
$$

Now, multiplying $(2.3)$ by $q(t)$, we get

$$
\begin{aligned}
0 & =w^{\Delta}(t) q(t)+p(t) q(t)+\mathscr{S}[q w, q r ; \alpha](t) \\
& \geq w^{\Delta}(t) q(t)+P(t) q(t)+\mathscr{S}[q w, q r ; \alpha](t) \\
& \geq w^{\Delta}(t) q(t)+w^{\sigma}(t) q^{\Delta}(t)+P(t) q(t)+\mathscr{S}[q w, q r ; \alpha](t) \\
& =(w q)^{\Delta}(t)+P(t) q(t)+\mathscr{S}[q w, q r ; \alpha](t)
\end{aligned}
$$

for $t \in[T, \infty)$. Hence, the function $v(t)=w(t) q(t)$ satisfies the generalized Riccati inequality $v^{\Delta}(t)+P(t) q(t)+\mathscr{S}[v, q r ; \alpha](t) \leq 0$ with

$$
\left\{\Phi^{-1}(q r)+\mu \Phi^{-1}(v)\right\}(t)=\Phi^{-1}(q)\left\{\Phi^{-1}(r)+\mu \Phi^{-1}(w)\right\}(t)>0
$$

for $t \in[T, \infty)$. Therefore, the equation

$$
\left[q(t) r(t) \Phi\left(x^{\Delta}\right)\right]^{\Delta}+q(t) P(t) \Phi\left(x^{\sigma}\right)=0
$$

is nonoscillatory by Proposition 2.2, and so (3.13) is nonoscillatory by Proposition 2.1 since $q(t) r(t) \leq r(t) \leq R(t)$.

Theorem 3.4. Assume that $q(t) \in C_{\mathrm{rd}}^{1}[a, \infty), 0<R(t) \leq r(t), p(t) \leq P(t), q(t) \geq 1$, and $q^{\Delta}(t) \geq 0$. Further, let

$$
\liminf _{t \rightarrow \infty} \int_{T}^{t} q(s) P(s) \Delta s \geq 0, \quad \liminf _{t \rightarrow \infty} \int_{T}^{t} q(s) P(s) \Delta s \not \equiv 0,
$$

for all large $T$, and

$$
\int_{a}^{\infty} R^{1-\beta}(s) \Delta s=\infty
$$

Then (1.2) is oscillatory implying that (3.13) is oscillatory.

Proof. Suppose, by a contradiction, that (3.13) is nonoscillatory. Then there exists a solution $x$ of (3.13) and $T \in[a, \infty)$ such that $x(t)>0$ and $x^{\Delta}(t)>0$ on $[T, \infty)$ by Lemma 2.5. Therefore, the function $v(t):=R(t) \Phi\left(x^{\Delta}(t) / x(t)\right)>0$ satisfies

$$
v^{\Delta}(t)+q(t) P(t)+\mathscr{S}[v, R ; \alpha](t)=0
$$

with $\left\{\Phi^{-1}(R)+\mu \Phi^{-1}(v)\right\}(t)>0$ on $[T, \infty)$. We have

$$
\frac{v^{\Delta}(t)}{q(t)} \geq \frac{v^{\Delta}(t) q(t)}{q^{2}(t)}-\frac{v(t) q^{\Delta}(t)}{q^{2}(t)}=\left(\frac{v(t)}{q(t)}\right)^{\Delta}
$$


at right-dense $t$, while

$$
\frac{v^{\Delta}(t)}{q(t)}=\frac{v^{\sigma}(t)}{\mu(t) q(t)}-\frac{v(t)}{\mu(t) q(t)} \geq \frac{v^{\sigma}(t)}{\mu(t) q^{\sigma}(t)}-\frac{v(t)}{\mu(t) q(t)}=\left(\frac{v(t)}{q(t)}\right)^{\Delta}
$$

at right-scattered $t$. Dividing (3.20) by $q(t)$ and using the above estimates, we get

$$
0=\frac{v^{\Delta}(t)}{q(t)}+P(t)+\frac{1}{q(t)} \mathscr{S}[v, R ; \alpha](t) \geq\left(\frac{v(t)}{q(t)}\right)^{\Delta}+p(t)+\mathscr{S}\left[\frac{v}{q}, \frac{R}{q} ; \alpha\right](t)
$$

for $t \in[T, \infty)$. Hence, the function $w(t)=v(t) / q(t)$ satisfies the inequality $w^{\Delta}(t)+p(t)+$ $\mathscr{Y}[w, R / q ; \alpha](t) \leq 0$ with $\left\{\Phi^{-1}(R / q)+\Phi^{-1}(w)\right\}>0$ for $t \in[T, \infty)$. Therefore, the equation

$$
\left[\frac{R(t)}{q(t)} \Phi\left(y^{\Delta}\right)\right]^{\Delta}+p(t) \Phi\left(y^{\sigma}\right)=0
$$

is nonoscillatory by Proposition 2.2. Now, since $R(t) / q(t) \leq R(t) \leq r(t)$, (1.2) is nonoscillatory by Proposition 2.1, a contradiction.

Remark 3.5. A closer examination of the proofs shows that the last two theorems can be improved in the following way (assuming the same conditions).

Theorem 3.3: (1.2) is nonoscillatory implying that (3.17) is nonoscillatory.

Theorem 3.4: (3.24) is oscillatory implying that (3.13) is oscillatory.

Our theorems then follow from the above by virtue of the Sturm-type comparison theorem.

We conclude the paper by the following application of Theorem 3.3.

Example 3.6. Let $\mathbb{T}=\mathbb{Z}$. Then $\mu(t) \equiv 1, f^{\Delta}(t)=\Delta f(t)$, and $\int_{a}^{b} f(t) \Delta t=\sum_{t=a}^{b-1} f(t)$. Further, let $r(t)=\left[(t+1)^{\beta-1}-t^{\beta-1}\right]^{1-\alpha}$ and

$$
p(t)=\frac{\gamma}{t(t+1)}+\frac{\lambda(-1)^{t}}{t}
$$

where $\gamma$ and $\lambda$ are real constants. It is easy to see that $p(t)$ changes sign for $\lambda \neq 0$. Moreover,

$$
\begin{aligned}
& \gamma-\lambda<t \sum_{s=t}^{\infty} p(s)<\gamma+\lambda, \\
& \sum_{s=0}^{t-1} r^{1-\beta}(s)=t^{\beta-1} \longrightarrow \infty
\end{aligned}
$$

as $t \rightarrow \infty$. In [17], it was proved (on general $\mathbb{T}$ ) that (1.2) is nonoscillatory provided that

$$
\begin{gathered}
\lim _{t \rightarrow \infty} \frac{\mu(t) r^{1-\beta}(t)}{\int_{a}^{t} r^{1-\beta}(s) \Delta s}=0, \\
-\frac{2 \alpha-1}{\alpha}\left(\frac{\alpha-1}{\alpha}\right)^{\alpha-1}<\liminf _{t \rightarrow \infty} \mathscr{A}(t) \leq \limsup _{t \rightarrow \infty} \mathscr{A}(t)<\frac{1}{\alpha}\left(\frac{\alpha-1}{\alpha}\right)^{\alpha-1},
\end{gathered}
$$


564 Comparison theorems for half-linear dynamic equations

where

$$
\mathscr{A}(t):=\left(\int_{a}^{t} r^{1-\beta}(s) \Delta s\right)^{\alpha-1} \int_{t}^{\infty} p(s) \Delta s .
$$

Hence, if $\gamma \geq \lambda>0$ and

$$
\gamma+\lambda<\frac{1}{\alpha}\left(\frac{\alpha-1}{\alpha}\right)^{\alpha-1}
$$

then (2.12) holds, (1.2) is nonoscillatory because of (3.26), and

$$
\mathscr{A}(t)=t \sum_{s=t}^{\infty} p(s)
$$

Consequently, equation

$$
\left[\left((t+1)^{\beta-1}-t^{\beta-1}\right)^{1-\alpha} \Phi\left(y^{\Delta}\right)\right]^{\Delta}+\left(\frac{\gamma q(t)}{t(t+1)}+\frac{\lambda(-1)^{t} q(t)}{t}\right) \Phi\left(y^{\sigma}\right)=0,
$$

where $q(t)$ is any nonincreasing sequence between 0 and 1 , is also nonoscillatory by Theorem 3.3.

\section{Acknowledgment}

This work was supported by the Grants 201/01/P041 and 201/01/0079 of the Czech Grant Agency. The author is grateful to Professor Ondřej Došlý for his helpful comments.

\section{References}

[1] R. P. Agarwal, Difference Equations and Inequalities, Monographs and Textbooks in Pure and Applied Mathematics, vol. 228, Marcel Dekker, New York, 2000.

[2] C. D. Ahlbrandt and A. C. Peterson, Discrete Hamiltonian Systems. Difference Equations, Continued Fractions, and Riccati Equations, Kluwer Texts in the Mathematical Sciences, vol. 16, Kluwer Academic Publishers, Dordrecht, 1996.

[3] M. Bohner and A. C. Peterson, Dynamic Equations on Time Scales. An Introduction with Applications, Birkhäuser Boston, Massachusetts, 2001.

[4] S. S. Cheng and W. T. Patula, An existence theorem for a nonlinear difference equation, Nonlinear Anal. 20 (1993), no. 3, 193-203.

[5] O. Došlý, Qualitative theory of half-linear second order differential equations, Math. Bohem. 127 (2002), no. 2, 181-195.

[6] Á. Elbert, A half-linear second order differential equation, Qualitative Theory of Differential Equations, Vol. I, II (Szeged, 1979), Colloq. Math. Soc. János Bolyai, vol. 30, North-Holland, Amsterdam, 1981, pp. 153-180.

[7] L. Erbe, A. C. Peterson, and P. Rehák, Comparison theorems for linear dynamic equations on time scales, J. Math. Anal. Appl. 275 (2002), no. 1, 418-438.

[8] D. H. Griffel, Applied Functional Analysis, Ellis Horwood Series in Mathematics and Its Applications, Ellis Horwood, Chichester, 1981.

[9] P. Hartman, Ordinary Differential Equations, John Wiley \& Sons, New York, 1973.

[10] S. Hilger, Analysis on measure chains-a unified approach to continuous and discrete calculus, Results Math. 18 (1990), no. 1-2, 18-56. 
[11] W. G. Kelley and A. C. Peterson, Difference Equations. An Introduction with Applications, Academic Press, Massachusetts, 1991.

[12] T. Kusano and N. Yoshida, Nonoscillation theorems for a class of quasilinear differential equations of second order, J. Math. Anal. Appl. 189 (1995), no. 1, 115-127.

[13] J. D. Mirzov, On some analogs of Sturm's and Kneser's theorems for nonlinear systems, J. Math. Anal. Appl. 53 (1976), no. 2, 418-425.

[14] L. Neidhart, Integration on measure chains, Proceedings of the 6th International Conference on Difference Equations and Applications (B. Aulbach, S. Elaydi, and G. Ladas, eds.), Taylor and Francis, Augsburg, Germany, in press.

[15] P. Řehák, Hartman-Wintner type lemma, oscillation, and conjugacy criteria for half-linear difference equations, J. Math. Anal. Appl. 252 (2000), no. 2, 813-827.

[16] - Oscillatory properties of second order half-linear difference equations, Czechoslovak Math. J. 51 (2001), no. 2, 303-321.

[17] Half-linear dynamic equations on time scales: IVP and oscillatory properties, Nonlinear Funct. Anal. Appl. 7 (2002), no. 3, 361-403.

[18] Comparison theorems and strong oscillation in the half-linear discrete oscillation theory, Rocky Mountain J. Math. 33 (2003), no. 1, 333-352.

[19] W. T. Reid, Sturmian Theory for Ordinary Differential Equations, Applied Mathematical Sciences, vol. 31, Springer-Verlag, New York, 1980.

[20] C. A. Swanson, Comparison and Oscillation Theory of Linear Differential Equations, Academic Press, New York, 1968.

Pavel Řehák: Mathematical Institute, Academy of Sciences of the Czech Republic, Žižkova 22, 61662 Brno, Czech Republic

E-mail address: rehak@math.muni.cz 


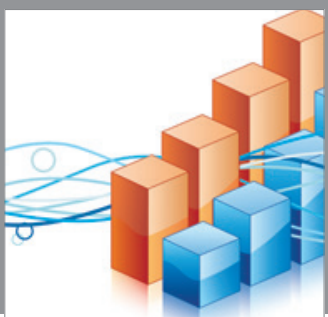

Advances in

Operations Research

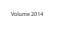

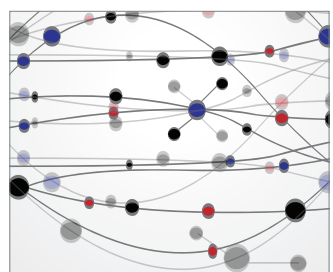

\section{The Scientific} World Journal
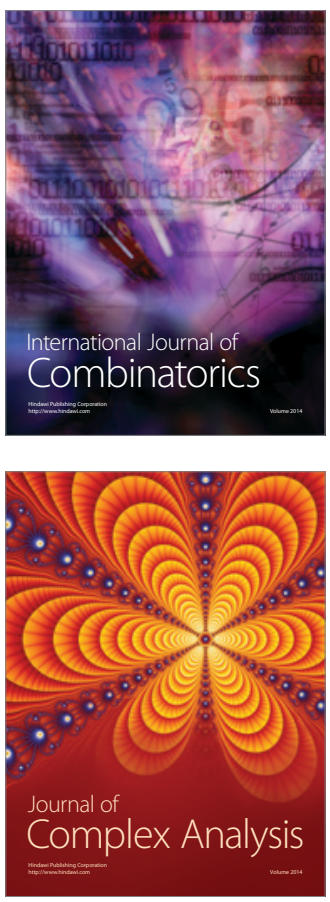

International Journal of

Mathematics and

Mathematical

Sciences
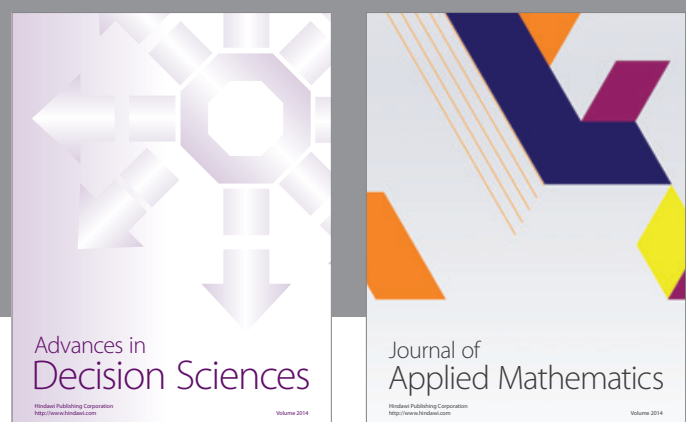

Journal of

Applied Mathematics
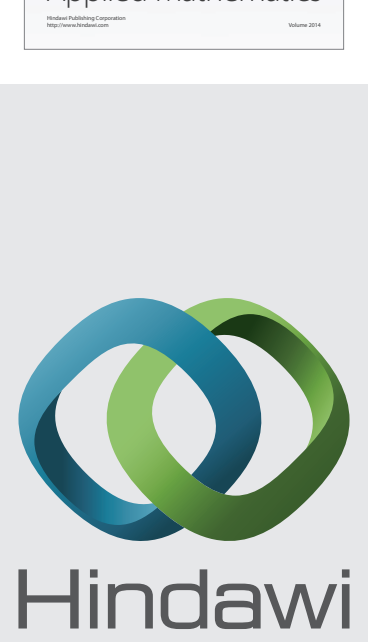

Submit your manuscripts at http://www.hindawi.com
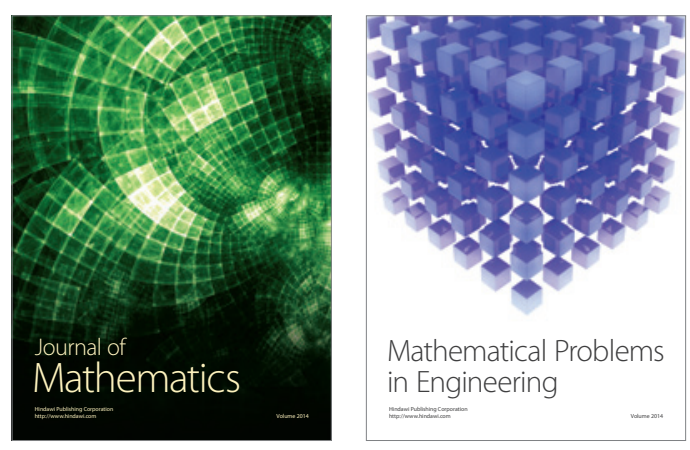

Mathematical Problems in Engineering
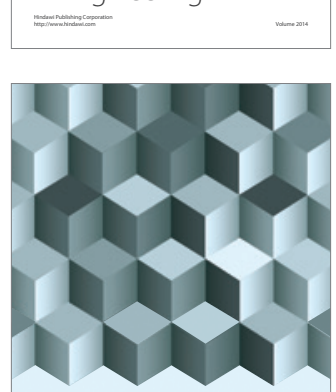

Journal of

Function Spaces
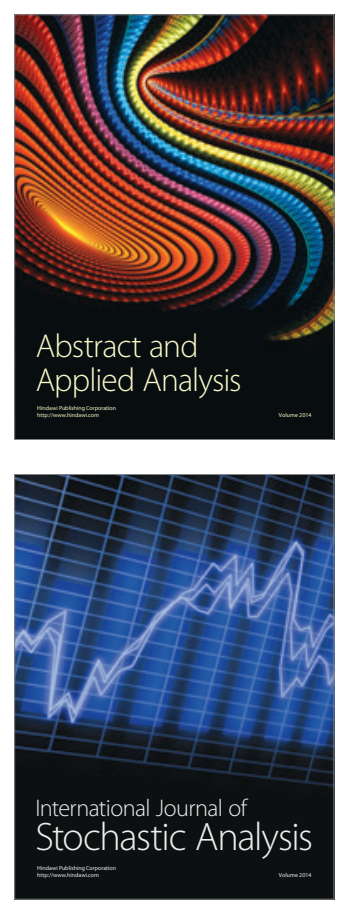

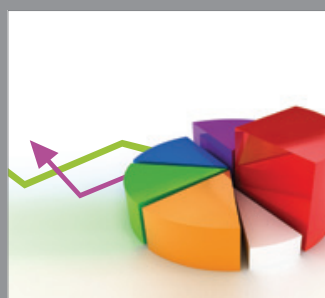

ournal of

Probability and Statistics

Promensencen
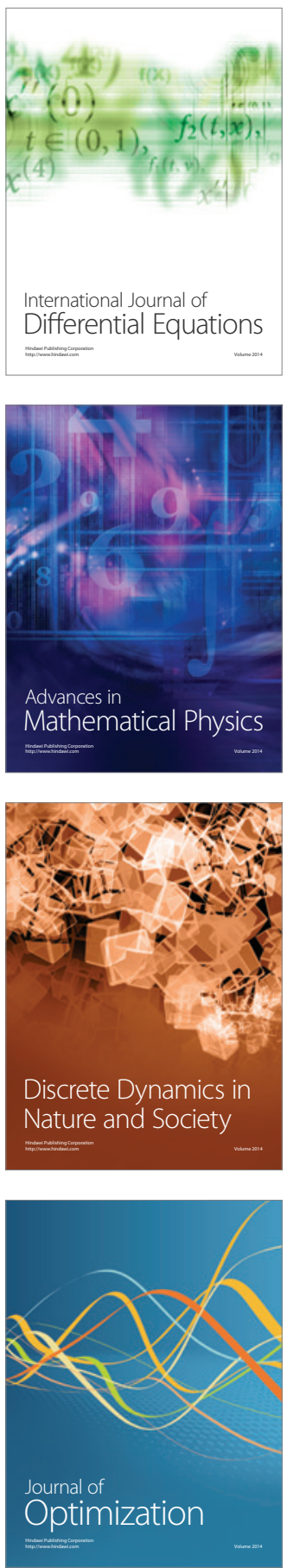